\title{
Immunogenicity and Safety of a Quadrivalent Meningococcal Serogroups A, C, W-135 and Y Tetanus Toxoid Conjugate Vaccine (MenACWY-TT) Administered to Adults Aged 56 Years and Older: Results of an Open-Label, Randomized, Controlled Trial
}

\author{
Ghassan Dbaibo • Nabil El-Ayoubi • \\ Soha Ghanem - Farah Hajar • Veronique Bianco • \\ Jacqueline M. Miller • Narcisa Mesaros \\ Published online: 14 March 2013 \\ (C) The Author(s) 2013. This article is published with open access at Springerlink.com
}

\begin{abstract}
Background The burden of invasive meningococcal disease is substantial in older adults in whom the case fatality rate is high. Travelers to regions with high rates of meningococcal disease, such as Hajj pilgrims, are at increased risk of meningococcal infection, and disease transmission from travelers to their close contacts has been documented. In younger individuals, meningococcal conjugate vaccines offer advantages over polysaccharide vaccines in terms of duration of protection and boostability, and induction of herd immune effects through reductions in nasopharyngeal carriage of meningococci. To date, few data are available evaluating meningococcal conjugate vaccine use in adults $>55$ years of age.

Objective To evaluate the immunogenicity and safety of quadrivalent meningococcal serogroups $\mathrm{A}, \mathrm{C}, \mathrm{W}-135$ and $\mathrm{Y}$ vaccine with all serogroups conjugated to tetanus toxoid
\end{abstract}

The study is registered at www.clinicaltrials.gov NCT01235975.

Electronic supplementary material The online version of this article (doi:10.1007/s40266-013-0065-0) contains supplementary material, which is available to authorized users.

G. Dbaibo $(\square) \cdot$ N. El-Ayoubi · S. Ghanem · F. Hajar

The Center for Infectious Diseases Research and the Department of Pediatrics and Adolescent Medicine, American University of Beirut, P.O. Box: 11-0236, Riad El Solh, Beirut 1107 2020,

Lebanon

e-mail: gdbaibo@aub.edu.lb

V. Bianco · N. Mesaros

GlaxoSmithKline Vaccines, Wavre, Belgium

J. M. Miller

GlaxoSmithKline Vaccines, King of Prussia, PA, USA
(MenACWY-TT, Nimenrix ${ }^{\mathrm{TM}}$, GlaxoSmithKline, Belgium) and a licensed quadrivalent polysaccharide vaccine (MenPS, Mencevax ${ }^{\mathrm{TM}}$ GlaxoSmithKline, Belgium) in adults $>55$ years of age.

Methods This was a phase IIIb, open-label, randomized (3:1), controlled study conducted at one study center in Lebanon. A total of 400 healthy adults between 56 and 103 years of age without previous MenPS or tetanus toxoid vaccination within the previous 5 years or meningococcal conjugate vaccination at any time previously were included. They received a single-dose vaccination with MenACWY-TT or MenPS with blood sampling before and 1 month after vaccination. The main outcome measures were serum bactericidal activity (rabbit complement source: $\mathrm{rSBA}$ ) vaccine response (VR) rate [rSBA titer of $\geq 1: 32$ in initially seronegative subjects (rSBA titer $<1: 8$ ); $\geq 4$-fold increase in subjects with pre-vaccination rSBA titers between 1:8 and 1:128, and $\geq 2$-fold increase in subjects with pre-vaccination rSBA titers $\geq 1: 128]$. The percentages of subjects with rSBA titers $\geq 1: 8$ and $\geq 1: 128$ and rSBA geometric mean titers (GMTs) were assessed. Solicited adverse events were recorded for 4 days following vaccination, and all other adverse events, including the incidence of new onset chronic diseases, were recorded for 31 days after vaccination.

Results One month after a single dose of MenACWY-TT, the rSBA VR rate in the MenACWY-TT group was $76.6 \%$ for serogroup A, $80.3 \%$ for serogroup C, $77.5 \%$ for serogroup W-135 and $81.9 \%$ for serogroup Y. VR rates in the MenPS group were 91.7, 84.8, 87.1 and $89.1 \%$, respectively. One month after vaccination, $\geq 93.2 \%$ of subjects in the MenACWY-TT group and $\geq 93.9 \%$ in the MenPS group had rSBA titers $\geq 1: 128$. In each group, 
GMTs increased by $\geq 13$-fold for each serogroup. rSBA VR and GMTs tended to be lower in subjects who were over 65 years compared to 56-65 years of age. Only $6.3 \%$ of MenACWY-TT recipients had anti-TT $\geq 0.1 \mathrm{IU} / \mathrm{ml}$ prior to vaccination, increasing to $28.1 \%$ post-vaccination. The rSBA GMTs were 1.9- to 4-fold higher in anti-TT responders. Each local and general solicited symptom was reported by no more than $3.0 \%$ of subjects in either group. No serious adverse events were considered vaccine related. Conclusion In adults 56 years of age and older, MenACWY-TT was immunogenic, with a vaccine response rate $\geq 76 \%$ and with $\geq 93 \%$ of subjects achieving rSBA titers $\geq 1: 128$ against all four serogroups after a single dose. MenACWY-TT induced low anti-TT concentrations in this population, which deserves further study.

\section{Background}

Invasive disease caused by Neisseria meningitidis is typically characterized by rapid onset with fulminant progression, resulting in death in around $10 \%$ of individuals despite appropriate antibiotics and supportive care [1]. The incidence of invasive meningococcal disease is highest in infants, with a second peak that occurs during adolescence [2]. Notwithstanding, a substantial proportion of the disease burden lies with adults: in the US approximately $43 \%$ of all invasive meningococcal disease cases were reported in adults aged 25 years and older (1998-2007 data) [2]. During the same observation period, $14 \%$ of cases occurred in the age group 65 years and older, with a case fatality rate of $23.8 \%$, the highest in any age group [2]. Similar trends are observed in other countries: in Australia $6 \%$ of all meningococcal cases in 2010 were in adults over 65 years of age, and $19 \%$ were in adults 45 years of age and older [3]. In England and Wales, around $10 \%$ of all meningococcal cases each year between 1993 and 2004 occurred in adults over 45 years of age [4]. These data point to a substantial disease burden in older adults, which is likely to become a greater public health concern as the global population ages.

Based on differences in the composition of the polysaccharide capsule, 12 meningococcal serogroups have been identified; however, 6 serogroups predominate as causes of invasive disease (serogroups A, B, C, W-135, X and $\mathrm{Y}$ ) [5]. In the Middle East, available information suggests that serogroups $\mathrm{A}$ and $\mathrm{W}-135$ are responsible for the majority of meningococcal invasive disease in the region. Serogroups A and W-135 are also responsible for major epidemics in Africa [1, 5], while serogroup $\mathrm{X}$ emerged more recently as a cause of local outbreaks in Africa [6-9]. By contrast, serogroups A, W-135 and X are infrequently identified in many Western countries, where serogroups $\mathrm{B}, \mathrm{C}$ and to a lesser degree $\mathrm{Y}$ predominate $[2,5]$.

Adults are at increased risk of meningococcal infection when they travel to meningococcal endemic regions [10]. A serogroup W-135 outbreak that occurred during the 2000 Hajj spread intercontinentally from pilgrims to their contacts $[11,12]$, and more recently, cases of $\mathrm{W}-135$ were identified in France among travellers who had returned from West Africa and their contacts [13]. These cases of intercontinental transmission highlight the relative ease by which meningococcal strains can be transmitted to immunologically naïve populations through global travel activity. Meningococcal vaccination against serogroups A, C, $\mathrm{W}-135$ and $\mathrm{Y}$ is now required prior to Hajj attendance for all pilgrims over 2 years of age [14].

Vaccination is the best strategy to prevent meningococcal disease. Quadrivalent serogroups A, C, W-135 and $\mathrm{Y}$ (ACWY) meningococcal polysaccharide vaccines are available for use in adults and children from the age of 2 years [15]. However, polysaccharide vaccines do not stimulate T-cell-dependent immune responses and thus do not induce long-lasting protection or immune memory, but may induce immune hyporesponsiveness, particularly for serogroup $\mathrm{C}$, on repeated administration [16, 17]. By contrast, meningococcal conjugate vaccines induce T-cellmediated immune responses that appear to be long-lived, with evidence of immune memory upon re-exposure [15]. Long-term protection against invasive meningococcal disease appears to be dependent on the presence of circulating antibody rather than on memory responses [18]. Antibody levels decrease over time after both meningococcal polysaccharide and meningococcal conjugate vaccination, and the duration of protection afforded by meningococcal conjugate vaccination is not yet known.

Conjugate vaccines also induce herd protection by decreasing the carriage rate in vaccine recipients, which was demonstrated in the UK after a mass vaccination program with conjugate serogroup C vaccines [19]. Herd protection is important in interrupting the transmission of meningococci. Meningococcal conjugate vaccines therefore have advantages in terms of duration of protection and boostability in those who require sustained protection. In adults, meningococcal ACWY (MenACWY) conjugate vaccines show comparable or improved immunogenicity versus polysaccharide vaccines [20-22]. However, few studies have evaluated conjugate vaccine use in adults over 55 years of age [20].

GlaxoSmithKline has developed a MenACWY vaccine with all serogroups conjugated to tetanus toxoid (MenACWY-TT, Nimenrix ${ }^{\mathrm{TM}}$ ), which is licensed for use in Europe as a single dose in individuals as of 1 year of age [23]. MenACWY-TT was immunogenic and well tolerated in clinical trials conducted in children, adolescents and 
adults up to age 55 years $[21,22,24-30]$. We conducted a phase IIIb, open-label, randomized, controlled study designed to evaluate the immunogenicity and safety of MenACWY-TT as compared to a licensed polysaccharide vaccine (Mencevax ${ }^{\mathrm{TM}}$ ACWY, GlaxoSmithKline Vaccines: MenPS) when administered as one dose to healthy adults aged 56 years and older.

\section{Methods}

\subsection{Study Design}

The study (113807, www.clinicaltrials.gov NCT01235975) was conducted in a single center in Lebanon between 30 November 2010 and 3 August 2011. Adults were enrolled and randomized 3:1 into two parallel groups. Subjects in the MenACWY-TT group received a single dose of MenACWY-TT, while subjects in the MenPS group received a single dose of licensed MenPS. A randomization list was generated at GlaxoSmithKline Belgium and was used to number the vaccines. Treatment allocation at the investigator site was performed using a central, web-based randomization system. A blocking scheme ensured that balance between treatments was maintained. The randomization algorithm used a minimization procedure accounting for center and age strata. The enrollment ensured a 2:1 allocation of the population across two age strata: $56-65$ years of age and $>65$ years of age.

This was an open study because the routes of administration of the study vaccine and the control vaccine were different.

The primary study objective was to evaluate the immunogenicity of MenACWY-TT as compared to MenPS in terms of serum bactericidal activity (rabbit complement source; rSBA) vaccine response (VR) rates 1 month after vaccination. A VR was defined as an rSBA titer of at least 1:32 in initially seronegative subjects (rSBA titer $<1: 8$ ), at least a four-fold increase in subjects with rSBA titers between 1:8 and 1:128 before vaccination and at least a two-fold increase in subjects with rSBA titers $\geq 1: 128$ before vaccination.

\subsection{Study Subjects}

Adults were to be at least 56 years of age at enrollment, and females were to be of non-child bearing potential. Subjects were not enrolled if they had received prior vaccination with MenPS or tetanus toxoid (TT) within the previous 5 years or with any meningococcal conjugate vaccine at any time previously. Subjects were also excluded if they had previously suffered from meningococcal disease, if they were immunosuppressed for any reason including chronic ( $>14$ days) immunosuppressant treatment, if they had a history of neurological disease, seizures or Guillain-Barre syndrome, or if they had active pulmonary, cardiovascular, hepatic or renal disease. Subjects who had received blood products within 3 months of vaccination or who had chronic alcohol consumption or drug abuse were also ineligible to participate.

\subsection{Study Vaccines}

One $0.5 \mathrm{ml}$ dose of MenACWY-TT contained $5 \mu \mathrm{g}$ each of meningococcal serogroup $\mathrm{A}, \mathrm{C}, \mathrm{W}-135$ and $\mathrm{Y}$ polysaccharide conjugated to a total of approximately $44 \mu \mathrm{g}$ TT. One $0.5-\mathrm{ml}$ dose of MenPS contained $50 \mu \mathrm{g}$ each of meningococcal serogroups $\mathrm{A}, \mathrm{C}, \mathrm{W}-135$ and $\mathrm{Y}$ polysaccharide. MenACWY-TT was administered intramuscularly and MenPS given subcutaneously into the upper arm on the non-dominant side.

\subsection{Immunogenicity Assessment}

Blood samples were collected from all subjects before and 1 month after vaccination and were tested for rSBA for each meningococcal serogroup as previously described [31]. The cutoff of the rSBA assay was a 1:8 dilution and was considered indicative of seroprotection [32, 33]. AntiTT antibodies were measured at each time point using an enzyme-linked immunosorbent assay (ELISA) [34], with an assay cutoff of $0.1 \mathrm{IU} / \mathrm{ml}$.

\subsection{Safety and Reactogenicity Assessment}

Specific local and general symptoms were recorded on diary cards for 4 days after vaccination. Symptom intensity of redness, swelling and fever was graded by millimeter of reaction and degrees Celsius of fever, respectively, and all other symptoms were graded by the subject using a predefined scale. Grade 3 redness and swelling were defined as diameter $>50 \mathrm{~mm}$, grade 3 fever as oral temperature $>39.5^{\circ} \mathrm{C}$, and for other symptoms, grade 3 was defined as preventing normal activity. All other adverse events including serious adverse events and new onset of chronic illness were recorded for 31 days after vaccination.

\subsection{Consent Procedures}

The study protocol was approved by the institutional review board (IRB) prior to study start. Written informed consent was obtained from each subject prior to enrollment.

Enrollment was hampered by the distance between home communities and the study center in Beirut. In response, the principal investigator proposed setting up a mobile unit to perform study visits by study personnel in local villages, as per protocol. A mobile unit guidance 
explaining the rationale for the mobile unit and the associated procedures was issued to the IRB for their information and the enrollment via the mobile unit started on the same day. Later, the IRB clarified that approval of the mobile unit was required. The IRB approved the mobile unit pending minor revisions a few days later. One hundred and two subjects were enrolled through the mobile unit prior to formal IRB mobile unit approval. All of these subjects were asked to re-consent. Twenty-nine subjects didn't re-consent (no subjects refused to re-consent because of an adverse event), and therefore these 29 subjects were eliminated from all statistical analyses.

\subsection{Statistical Analyses}

As this is the first study to investigate the immunogenicity of MenACWY-TT in a population of older adults, this exploratory study was designed as a descriptive analysis. The primary analysis of immunogenicity was done on the according-to-protocol (ATP) cohort for immunogenicity, which included subjects who had complied with all protocoldefined procedures and who had data available for at least one immunogenicity endpoint. Percentages of subjects with rSBA vaccine response and with titers/concentrations above threshold values, with exact $95 \%$ CIs, were calculated. The effect of age at vaccination and of meningococcal vaccination history was investigated in exploratory analyses.

The target enrollment was set at 400 subjects randomized 3:1 to the MenACWY-TT group or MenPS group in order to have 360 evaluable subjects (270 subjects in MenACWY-TT group and 90 subjects in MenPS group), assuming a $10 \%$ drop out of subjects throughout the study. With 270 evaluable subjects in the MenACWY-TT group and assuming a VR rate of $80 \%$, the exact $95 \% \mathrm{CI}$ around the estimate would be $74.7 ; 84.6$. With 90 evaluable subjects in the MenPS group, the $95 \% \mathrm{CI}$ around the assumed VR rate of $80 \%$ would be $70.2 ; 87.7$.

Exploratory analyses were used to highlight potential differences between groups if the asymptotic standardized $95 \%$ CI for the group difference in the percentage of subjects reaching specified immunological cutoffs did not contain the value ' 0 ' or if the $95 \% \mathrm{CI}$ for the geometric mean titer (GMT) ratio (ANCOVA model using the prevaccination logarithm 10 transformation of the titers/ concentrations, the vaccine group, age strata and meningococcal vaccination history as covariates) between groups did not contain the value ' 1 '. Multiple comparisons were done without adjustment for multiplicity, and there is a risk that statistically significant differences may have occurred by chance alone. Thus, any statistically significant differences between groups should be interpreted cautiously. Exploratory analyses for statistical differences were performed on the cohort including all subjects; these analyses were not performed per age strata because of the limited available sample size per stratum.

The primary analysis of safety was done on the total vaccinated cohort, comprising all vaccinated subjects with safety data available.

Statistical analyses were performed using SAS $^{\circledR}$ software version 9.2 (SAS Institute Inc., Cary, NC, USA) and ProcStatXact 8.1.

\section{Results}

\subsection{Study Subjects}

Four hundred adults whose age ranged between 56 and 103 years were enrolled in the study. Of these, 2 subjects were not vaccinated, and 29 were excluded from all statistical analyses (see Sect. 2). Thus, there were 369 subjects in the total vaccinated cohort, of which $260(70 \%)$ were eligible for inclusion in ATP analyses (Fig. 1). More females $(68.3 \%)$ than males $(31.7 \%)$ were enrolled. The two treatment groups were comparable in terms of demographic characteristics (Table 1).

One subject (an 84-year-old subject in the MenPS group) withdrew from the study because of a serious adverse event. This subject suffered a cerebrovascular accident and died 21 days after vaccination. The event was considered unrelated to vaccination by the investigator.

\subsection{Immunogenicity}

After a single dose of MenACWY-TT, the VR rate in the MenACWY-TT group was $76.6 \%$ for serogroup A, $80.3 \%$ for serogroup C, $77.5 \%$ for serogroup W-135 and $81.9 \%$ for serogroup Y (Table 2). VR rates in the MenPS group were $91.7,84.8,87.1$ and $89.1 \%$, respectively.

One month after vaccination, at least $97.4 \%$ in the MenACWY-TT group and at least $95.5 \%$ in the MenPS group had rSBA titers $\geq 1: 8$ (Table 3 ). The percentage with rSBA $\geq 1: 128$ was at least $93.2 \%$ in the MenACWY-TT group and at least $93.9 \%$ in the MenPS group. In each group, GMTs increased by at least 13 -fold for each serogroup (Table 3).

Exploratory analyses did not detect any statistically significant differences between groups in terms of the percentage of subjects who reached the 1:8 and 1:128 thresholds after vaccination. However, these analyses suggested that the magnitude of the response was statistically significantly lower in MenACWY-TT recipients than in MenPS recipients in terms of VR rate for serogroup A and rSBA GMTs for serogroups A and C. VR rates in individuals with pre-existing rSBA titers $\geq 1: 128$ tended to be lower for each serogroup in MenACWY-TT recipients 
Fig. 1 Subject flow through the study. ATP according to protocol, $S A E$ serious adverse event. *Twenty-nine subjects (randomized and vaccinated) who were enrolled via the mobile unit prior to approval and who did not re-consent after mobile unit approval by the institutional review board

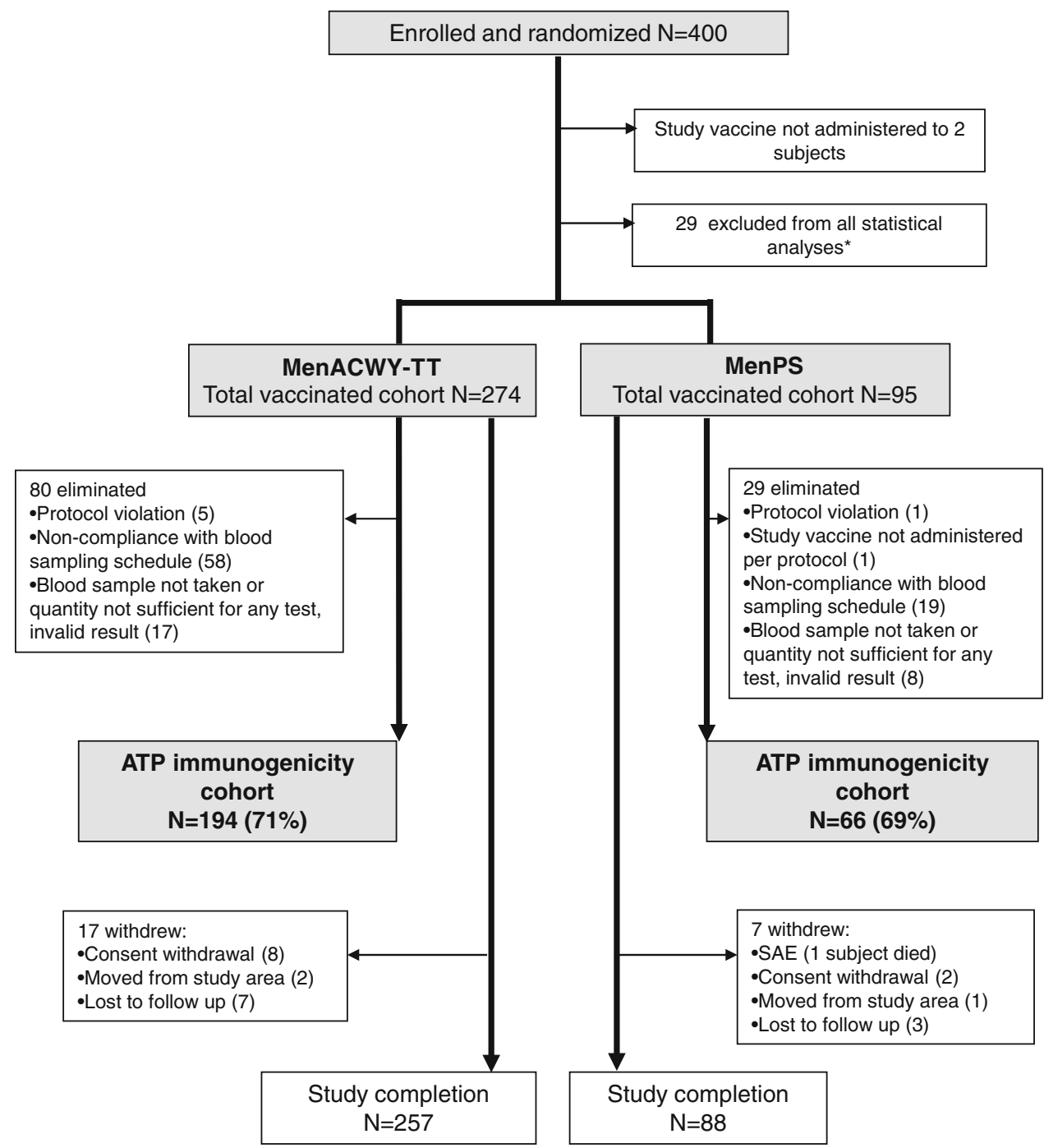

(65.7-72.6\%) than in MenPS recipients (75.9-91.3\%), while the majority of initially seronegative subjects in both groups demonstrated VRs to each serogroup after vaccination (for serogroup A, $93.3 \%$ in the MenACWY-TT group and $100 \%$ in the MenPS group; for serogroup C, 96.3 and $90.9 \%$; for $\mathrm{W}-135,88.7$ and $86.4 \%$; and for $\mathrm{Y}$, $100 \%$ in both groups).

\subsection{Effect of Age and Previous Meningococcal} Polysaccharide Vaccination on the Immune Response

VR rates tended to be lower in subjects who were $>65$ years of age at the time of vaccination, compared to younger (56- to 65-year-old) subjects (Table 2). This trend was more pronounced in MenPS recipients for serogroups A, C and Y. Possible differences between groups were not statistically tested as these tests are not powered to do so, because of the low numbers of subjects in each subgroup.
The percentage of subjects with rSBA titers $\geq 1: 128$ and the rSBA GMT were within the same range in the 56-65 year and >65 year subgroups, although the small number of subjects in each group means that conclusions cannot be drawn (Supplementary table 1).

Approximately one-quarter of subjects had a history of having received a meningococcal polysaccharide vaccine more than 5 years previously (Table 1). Prior to vaccination, the percentage of subjects with rSBA $\geq 1: 128$ was between 57.5 and $60.7 \%$ for serogroup A, 43.9 and $45.3 \%$ for serogroup C, 48.4 and $52.1 \%$ for serogroup $\mathrm{W}-135$ and 62.4 and $71.9 \%$ for serogroup Y (Table 3). The percentage of subjects with pre-vaccination titers $\geq 1: 128$ and rSBA GMTs for serogroups A and C, but not W-135 or Y, tended to be higher in subjects with a history of meningococcal vaccination than in unvaccinated subjects (Supplementary table 2). In the MenACWY-TT group, the post-vaccination rSBA GMTs in subjects who had not received a meningococcal dose previously were in the same range as in 
Table 1 Summary of demographic characteristics (total vaccinated cohort)

\begin{tabular}{|c|c|c|}
\hline Characteristic & MenACWY-TT $N=274$ & MenPS $N=95$ \\
\hline \multicolumn{3}{|l|}{ Age (years) } \\
\hline Mean (SD) & $64.1(7.22)$ & $64.3(7.39)$ \\
\hline Range & $56-103$ & $56-86$ \\
\hline \multicolumn{3}{|c|}{ Age stratum (years) $n(\%)$} \\
\hline $56-65$ & $184(67.2)$ & $63(66.3)$ \\
\hline$>65$ & $90(32.8)$ & $32(33.7)$ \\
\hline \multicolumn{3}{|l|}{ Gender $n(\%)$} \\
\hline Female & $182(66.4)$ & $70(73.7)$ \\
\hline Male & $92(33.6)$ & $25(26.3)$ \\
\hline \multicolumn{3}{|c|}{ Geographic ancestry $n(\%)$} \\
\hline Arabic/North African & $274(100)$ & $95(100)$ \\
\hline \multicolumn{3}{|c|}{ Meningococcal vaccination history $^{\mathrm{a}} n(\%)$} \\
\hline Unknown & $2(0.7)$ & $2(2.1)$ \\
\hline 0 & $210(76.6)$ & $68(71.6)$ \\
\hline 1 & $62(22.6)$ & $25(26.3)$ \\
\hline 2 & $0(0.0)$ & $0(0.0)$ \\
\hline
\end{tabular}

$N$ total number of subjects, $n / \%$ number/percentage of subjects in a given category, $S D$ standard deviation

${ }^{\text {a }}$ Number of previous meningococcal polysaccharide vaccinations. Note that previous meningococcal vaccines were received more than 5 years prior to enrollment

subjects who had received a prior meningococcal vaccination, although lower point values for rSBA-W-135 titers were observed in those previously vaccinated: for serogroup A, 1,372.0 [95 \% CI 1,088.3; 1,729.6] versus $1,726.4$ $[1,067.5 ; 2,792.0]$; for serogroup $\mathrm{C}, 2,584.3[1,836.2$;
$3,637.2]$ versus $2,412.1[1,504.7 ; 3,866.7]$; for serogroup $\mathrm{W}-135,1,791.7[1,369.7 ; 2,343.7]$ versus $756.2[406.2$; 1,407.7]; and for serogroup Y, 2,664.0 [2,107.0; 3,368.3] versus $2,219.6[1,324.1 ; 3,720.6]$. In the MenPS group, the rSBA GMTs were: for serogroup A, 2,640.3 [1,768.5; $3,941.9]$ versus $3,968.1$ [2,248.5; 7,003.0]; for serogroup C, $5,054.1 \quad[2,661.9 ; 9,595.9]$ versus $4,712.0 \quad[1,483.6$; 14,965.4]; for serogroup W-135, 2,217.4 [1,363.6; 3,606.0] versus $1,325.9$ [334.1; 5,261.7]; and for serogroup $\mathrm{Y}$, $4,370.4 \quad[2,756.7 ; 6,928.6]$ versus $3,710.3 \quad[2,042.1$; 6,741.0]. Due to the low numbers of subjects in each subgroup, comparisons between groups are not reliable (Supplementary table 2).

\subsection{Response to Tetanus Toxoid}

Few subjects had anti-TT antibodies $\geq 0.1 \mathrm{IU} / \mathrm{ml}$ prior to vaccination (12 subjects, $6.3 \%$ in the MenACWY-TT group and 6 subjects, $9.1 \%$ in the MenPS group). The antiTT response after MenACWY-TT vaccination was low (Table 4).

A post-hoc analysis assessed rSBA GMTs in subjects according to pre- and post-vaccination anti-TT antibody status in the MenACWY-TT group. In adults with anti-TT antibodies $\geq 0.1 \mathrm{IU} / \mathrm{ml}$ prior to vaccine, post-vaccination $\mathrm{rSBA}$ GMTs after MenACWY-TT were as high or higher than those observed in the MenPS group for serogroups C, W-135 and Y, but not for serogroup A (rSBA GMT 2,479.8 [95\% CI $1,211.4 ; 5,076.2]$ for serogroup A, 7,492.7 [2,069.9; 27,121.6] for serogroup C, 3,250.5 [521.4; 20,263.8] for serogroup W-135, and 3,992.0 [1,028.7; 15,491.2] for serogroup Y, Supplementary table 3). Furthermore, the post-vaccination

Table 2 Percentage of subjects with a vaccine response 1 month after vaccination: all subjects and by age strata (ATP cohort for immunogenicity)

\begin{tabular}{|c|c|c|c|c|c|c|c|c|c|c|}
\hline \multirow[t]{2}{*}{ Serogroup } & \multirow[t]{2}{*}{ Group } & \multicolumn{3}{|c|}{ All subjects } & \multicolumn{3}{|c|}{ 56- to 65 -year-olds } & \multicolumn{3}{|c|}{$>65$-year-olds } \\
\hline & & $N$ & $n$ & $\%[95 \% \mathrm{CI}]$ & $N$ & $n$ & $\%[95 \% \mathrm{CI}]$ & $N$ & $n$ & $\%[95 \% \mathrm{CI}]$ \\
\hline \multirow[t]{2}{*}{ A } & MenACWY-TT & 175 & 134 & $76.6 *[69.6 ; 82.6]$ & 119 & 93 & $78.2[69.6 ; 85.2]$ & 56 & 41 & $73.2[59.7 ; 84.2]$ \\
\hline & MenPS & 60 & 55 & $91.7[81.6 ; 97.2]$ & 37 & 37 & $100[90.5 ; 100]$ & 23 & 18 & $78.3[56.3 ; 92.5]$ \\
\hline \multirow[t]{2}{*}{$\mathrm{C}$} & MenACWY-TT & 188 & 151 & $80.3[73.9 ; 85.7]$ & 128 & 106 & $82.8[75.1 ; 88.9]$ & 60 & 45 & $75.0[62.1 ; 85.3]$ \\
\hline & MenPS & 66 & 56 & $84.8[73.9 ; 92.5]$ & 41 & 38 & $92.7[80.1 ; 98.5]$ & 25 & 18 & $72.0[50.6 ; 87.9]$ \\
\hline \multirow[t]{2}{*}{$\mathrm{W}-135$} & MenACWY-TT & 187 & 145 & $77.5[70.9 ; 83.3]$ & 127 & 102 & $80.3[72.3 ; 86.8]$ & 60 & 43 & $71.7[58.6 ; 82.5]$ \\
\hline & MenPS & 62 & 54 & $87.1[76.1 ; 94.3]$ & 38 & 33 & 86.8 [71.9; 95.6] & 24 & 21 & $87.5[67.6 ; 97.3]$ \\
\hline \multirow[t]{2}{*}{ Y } & MenACWY-TT & 188 & 154 & $81.9[75.7 ; 87.1]$ & 128 & 103 & $80.5[72.5 ; 86.9]$ & 60 & 51 & $85.0[73.4 ; 92.9]$ \\
\hline & & 64 & 57 & $89.1[78.8 ; 95.5]$ & 39 & 37 & 94.9 [82.7; 99.4] & 25 & 20 & 80.0 [59.3; 93.2] \\
\hline
\end{tabular}

Vaccine response defined as: post-vaccination rSBA titer $\geq 1: 32$ in initially seronegative subjects; four-fold increase in initially seropositive subjects with rSBA titer 1:8 and $<1: 128$; two-fold increase in initially seropositive subjects with rSBA titer $\geq 1: 128$

$A T P$ according-to-protocol, $N$ number of subjects with both pre- and post-vaccination results available, $n / \%$ number/percentage of responders; $95 \%$ CI $95 \%$ confidence interval, $r S B A$ rabbit complement source

* Statistically significant difference between the MenACWY-TT and MenPS groups (exploratory analysis) 
Table 3 Percentage of subjects with rSBA titers $\geq 1: 8$ and $\geq 1: 128$ and GMTs (ATP cohort for immunogenicity)

\begin{tabular}{|c|c|c|c|c|c|c|c|c|}
\hline \multirow[t]{2}{*}{ Serogroup } & \multirow[t]{2}{*}{ Group } & \multirow[t]{2}{*}{ Time point } & \multirow[t]{2}{*}{$N$} & \multicolumn{2}{|c|}{$\geq 1: 8$} & \multicolumn{2}{|c|}{$\geq 1: 128$} & \multirow[t]{2}{*}{ GMT $[95 \% \mathrm{CI}]$} \\
\hline & & & & $n$ & $\%[95 \% \mathrm{CI}]$ & $n$ & $\%[95 \% \mathrm{CI}]$ & \\
\hline \multirow[t]{4}{*}{ A } & \multirow[t]{2}{*}{ MenACWY-TT } & Pre & 181 & 136 & $75.1[68.2 ; 81.3]$ & 104 & $57.5[49.9 ; 64.8]$ & $108.3[77.9 ; 150.5]$ \\
\hline & & Post & 186 & 185 & $99.5[97.0 ; 100]$ & 177 & $95.2[91.0 ; 97.8]$ & $1,442.3 *[1,174.4 ; 1,771.3]$ \\
\hline & \multirow[t]{2}{*}{ MenPS } & Pre & 61 & 43 & $70.5[57.4 ; 81.5]$ & 37 & $60.7[47.3 ; 72.9]$ & $102.1[55.2 ; 188.7]$ \\
\hline & & Post & 65 & 65 & $100[94.5 ; 100]$ & 63 & $96.9[89.3 ; 99.6]$ & $2,840.1[2,062.3 ; 3,911.1]$ \\
\hline \multirow[t]{4}{*}{$\mathrm{C}$} & \multirow[t]{2}{*}{ MenACWY-TT } & Pre & 190 & 135 & $71.1[64.0 ; 77.4]$ & 86 & $45.3[38.0 ; 52.6]$ & $71.5[51.1 ; 100.1]$ \\
\hline & & Post & 192 & 192 & $100[98.1 ; 100]$ & 179 & $93.2[88.7 ; 96.3]$ & $2,498.6^{*}[1,887.0 ; 3,308.2]$ \\
\hline & \multirow[t]{2}{*}{ MenPS } & Pre & 66 & 44 & $66.7[54.0 ; 77.8]$ & 29 & $43.9[31.7 ; 56.7]$ & $73.8[38.0 ; 143.6]$ \\
\hline & & Post & 66 & 65 & $98.5[91.8 ; 100]$ & 62 & $93.9[85.2 ; 98.3]$ & $4,815.1[2,827.0 ; 8,201.2]$ \\
\hline \multirow[t]{4}{*}{$\mathrm{W}-135$} & \multirow[t]{2}{*}{ MenACWY-TT } & Pre & 188 & 135 & $71.8[64.8 ; 78.1]$ & 98 & $52.1[44.7 ; 59.5]$ & $84.7[61.1 ; 117.3]$ \\
\hline & & Post & 193 & 188 & $97.4[94.1 ; 99.2]$ & 183 & $94.8[90.7 ; 97.5]$ & $1,454.0[1,130.5 ; 1,870.1]$ \\
\hline & \multirow[t]{2}{*}{ MenPS } & Pre & 62 & 40 & $64.5[51.3 ; 76.3]$ & 30 & $48.4[35.5 ; 61.4]$ & $68.5[37.4 ; 125.3]$ \\
\hline & & Post & 66 & 63 & $95.5[87.3 ; 99.1]$ & 62 & $93.9[85.2 ; 98.3]$ & $1,838.4[1,134.6 ; 2,978.9]$ \\
\hline \multirow[t]{4}{*}{$\mathrm{Y}$} & \multirow[t]{2}{*}{ MenACWY-TT } & Pre & 189 & 148 & $78.3[71.7 ; 84.0]$ & 118 & $62.4[55.1 ; 69.4]$ & $137.6[100.7 ; 187.9]$ \\
\hline & & Post & 193 & 193 & $100[98.1 ; 100]$ & 187 & $96.9[93.4 ; 98.9]$ & $2,547.0[2,059.6 ; 3,149.8]$ \\
\hline & \multirow[t]{2}{*}{ MenPS } & Pre & 64 & 55 & $85.9[75.0 ; 93.4]$ & 46 & $71.9[59.2 ; 82.4]$ & $217.4[131.7 ; 358.9]$ \\
\hline & & Post & 66 & 66 & $100[94.6 ; 100]$ & 65 & $98.5[91.8 ; 100]$ & $3,931.6[2,726.1 ; 5,670.2]$ \\
\hline
\end{tabular}

$A T P$ according-to-protocol, $G M T$ geometric mean antibody titer calculated on all subjects, $N$ number of subjects with available results, $n / \%$ number/percentage of subjects with titer within the specified range, $95 \%$ CI $95 \%$ confidence interval, Pre Pre-vaccination, Post 1 month postvaccination, $r S B A$ rabbit complement source

* Statistically significant difference between the MenACWY-TT and MenPS groups (exploratory analysis)

Table 4 Percentage of subjects with anti-TT concentrations $\geq 0.1$ and $1 \mathrm{IU} / \mathrm{ml}$ and GMCs (ATP cohort or immunogenicity)

\begin{tabular}{|c|c|c|c|c|c|c|c|}
\hline \multirow[t]{2}{*}{ Group } & \multirow[t]{2}{*}{ Timing } & \multirow[t]{2}{*}{$N$} & \multicolumn{2}{|c|}{$\geq 0.1 \mathrm{lU} / \mathrm{ml}$} & \multicolumn{2}{|c|}{$\geq 1 \mathrm{lU} / \mathrm{ml}$} & \multirow[t]{2}{*}{ GMC $[95 \% \mathrm{CI}]$} \\
\hline & & & $n$ & $\%[95 \% \mathrm{CI}]$ & $n$ & $\%[95 \% \mathrm{CI}]$ & \\
\hline \multirow[t]{2}{*}{ MenACWY-TT } & Pre & 192 & 12 & $6.3[3.3 ; 10.7]$ & 3 & $1.6[0.3 ; 4.5]$ & $0.058[0.053 ; 0.063]$ \\
\hline & Post & 192 & 54 & $28.1[21.9 ; 35.1]$ & 29 & $15.1[10.4 ; 21.0]$ & $0.137[0.104 ; 0.180]$ \\
\hline \multirow[t]{2}{*}{ MenPS } & Pre & 66 & 6 & $9.1[3.4 ; 18.7]$ & 1 & $1.5[0.0 ; 8.2]$ & $0.060[0.051 ; 0.070]$ \\
\hline & Post & 66 & 6 & $9.1[3.4 ; 18.7]$ & 1 & $1.5[0.0 ; 8.2]$ & $0.060[0.051 ; 0.071]$ \\
\hline
\end{tabular}

ATP according-to-protocol, GMC geometric mean antibody concentration calculated on all subjects, $N$ number of subjects with available results, $n / \%$ number/percentage of subjects with concentration within the specified range, $95 \%$ CI $95 \%$ confidence interval, Pre Pre-vaccination, Post 1 month post-vaccination, $T T$ tetanus toxoid

rSBA GMTs were between 1.9- and 4-fold higher in subjects who responded to TT: That is, the point values of the rSBA GMT for each serogroup was higher (no statistical test performed because of the low number of subjects) in subjects with post-vaccination anti-TT concentrations $\geq 1.0 \mathrm{IU} / \mathrm{ml}$ (3,581.8 [95 \% CI 2,211.4; 5,801.4] for serogroup A, 3,891.4 $[1,605.7 ; 9,430.6]$ for serogroup C, $3,750.9$ [1,985.8; 7,084.8] for serogroup W-135 and 7,600.5 [4,588.7; 12,589.3] for serogroup $\mathrm{Y}$ ) and was lower in subjects in whom the anti-TT concentration remained $<0.1 \mathrm{IU} / \mathrm{ml}$ after vaccination $(1,147.8$ [891.5; 1,477.7] for serogroup A, 2,101.0 [1,510.1; 2,923.0] for serogroup C, $1,045.1$ [788.0; 1,385.9] for serogroup W-135 and 1,831.1 [1,441.2; 2,326.6] for serogroup Y) (Supplementary table 3).

\subsection{Reactogenicity and Safety}

Incidences of local and general solicited symptoms were very low in both groups. In the MenACWY-TT group pain was reported by six subjects $(2.3 \%$ [95 \% CI $0.8 ; 4.9])$ and redness and swelling each by three subjects $(1.1 \%[0.2$; 3.3]). No local symptoms were reported by subjects in the MenPS group. No local symptoms of grade 3 intensity were reported in either group.

Fatigue was reported by five subjects in the MenACWY-TT group (1.9\% [95\% CI 0.6; 4.3]) and no subjects in the MenPS group. Headache was reported by eight subjects $(3.0 \%[1.3 ; 5.9])$ in the MenACWY-TT group and by two subjects $(2.2 \%[0.3 ; 7.9])$ in the MenPS group. 
Fever (defined as axilliary temperature $\geq 37.5^{\circ} \mathrm{C}$ ) was reported by six subjects $(2.3 \%[0.8 ; 4.9])$ in the MenACWY-TT group and by one subject $(1.1 \%[0.0 ; 6.1])$ in the MenPS group. All fever episodes were $\leq 38.0^{\circ} \mathrm{C}$ except one, which was $>38.0^{\circ} \mathrm{C}$ and one $>38.5^{\circ} \mathrm{C}$ in the MenACWT-TT group. No subject in either group reported gastrointestinal symptoms during the solicited follow-up period.

No grade 3 local or general symptoms were reported by either group, and no solicited symptom led to a medicallyattended visit. On observing the low incidences of adverse reactions after vaccination, the investigator re-questioned the subjects before the database was unblinded and the statistical analysis performed, and additional adverse events were not identified.

One serious adverse event was reported during the study (reported above). No cases of new onset of chronic disease were reported during the 31-day follow-up period.

\section{Discussion}

This is the first study to evaluate quadrivalent MenACWYTT in an elderly population. MenACWY-TT was immunogenic in this age group, with at least $93.2 \%$ of vaccinees achieving rSBA titers $\geq 1: 128$ after vaccination, at least a 13.3-fold increase in post-vaccination rSBA GMTs, and a VR observed in at least $76.6 \%$ of subjects.

In contrast with previous studies conducted in children, adolescents and adults, we observed lower responses in the MenACWY-TT group compared to the MenPS group in terms of VR (serogroup A) and GMTs (serogroups A and C), mainly because of a lower response in subjects with high pre-existing rSBA titers $(\geq 1: 128)$, indicative of previous vaccination or exposure, who received MenACWY-TT. Immune hyporesponsiveness, in which the individual fails to mount an immune response after booster vaccination of at least the same, or of a greater magnitude, than that achieved after primary vaccination [16], has been well described in all age groups following repeated vaccination with serogroup $\mathrm{C}$ polysaccharide vaccine [17, 35]. Available data from studies in adolescents and young adults using MenACWY-TT or MenACWY-DT (diphtheria toxoid) show attenuated responses to all serogroups in subjects previously vaccinated with polysaccharide vaccines compared to vaccine-naïve subjects receiving their first conjugate vaccine dose [36-38]. This suggests that conjugate meningococcal vaccines only partially reverse immune hyporesponsiveness induced by prior meningococcal polysaccharide vaccination. Nevertheless, immune responses following meningococcal conjugate vaccination in previously vaccinated subjects were consistently high, with seroprotective rSBA levels achieved in the majority of subjects [36-38]. We observed comparable responses regardless of previous meningococcal vaccination in the MenACWY-TT group, except for the lower point values for serogroup W-135 rSBA titers. This study was not designed to evaluate booster responses in previously primed individuals, and we are unable to reach conclusions on the nature of the responses observed. Further studies are needed to define how meningococcal polysaccharide vaccination influences subsequent conjugate vaccination in adults.

Our study is one of the few that provide information on the immunogenicity of meningococcal conjugate vaccination in the elderly. ACWY-CRM 197 was observed to be immunogenic in older individuals in a study that included a cohort of previously unvaccinated subjects between 55 and 65 years of age with SBA titers (human complement source) that were higher following conjugate vaccination than after meningococcal polysaccharide vaccination [20]. MenACWY-TT was also immunogenic in all four vaccine serogroups in subjects whose age ranged between 56 and 103 years. Our study comprised more females than males, which is typical of the gender distribution of this age group [39].

Conjugate vaccines using TT as carrier are typically highly immunogenic, in part related to T-cell help induced by the TT carrier [40]. We observed a very low percentage of subjects with anti-TT concentrations $\geq 0.1 \mathrm{IU} / \mathrm{ml}$ prior to vaccination and low anti-TT responses to a single dose of MenACWY-TT, consistent with vaccination practices in Lebanon where no tetanus booster is routinely recommended after childhood. Similarly low anti-TT seroprotection rates have been reported in other countries: In Turkey, only $15.4 \%$ of nursing home residents (mean age 71 years) had anti-TT antibodies $\geq 0.1 \mathrm{IU} / \mathrm{ml}$ by ELISA [41]; in Egypt only $9.7 \%$ of elderly individuals had anti-TT antibodies $\geq 0.15 \mathrm{IU} / \mathrm{ml}$ by ELISA [42], while in Spain, only $7.7 \%$ of individuals over 70 years of age had protective titers by hemagglutination [43]. This is in contrast to studies done with MenACWY-TT in other adult populations where pre-vaccination anti-TT concentrations were higher (e.g., $51.5 \%$ with anti-TT $\geq 0.1 \mathrm{IU} / \mathrm{ml}$ prior to vaccination in an Asian adult population aged 18-55 years, including adults from Lebanon [21]). The TT component of MenACWY-TT was highly immunogenic in that study (percentage of participants with anti-TT $\geq 0.1 \mathrm{IU} / \mathrm{ml}$ increased to $79.4 \%$ ) [21, 30]. Although vaccination records were not available, the low responses observed in our study may reflect a primary immune response, as observed following a single dose of TT in adults with an unknown or distant history of TT vaccination [44]. The absence of preexisting immunity to TT or a vaccine response to the TT component of MenACWY-TT may have reduced the available T-cell help, resulting in lower rSBA responses than previously observed, appearing to particularly affect 
serogroups A and C. This is supported by the observation that rSBA responses were 1.9- to 4-fold higher in individuals who mounted a booster response to TT (concentrations $\geq 1.0 \mathrm{IU} / \mathrm{ml}$ after vaccination) compared with those in whom the magnitude of the post-vaccination anti-TT concentration was lower. Immunogenicity of conjugate vaccines using DT and $\mathrm{CRM}_{197}$ carrier proteins is known to need prior DT priming to generate optimal responses [40]. Our results support the immunogenicity of MenACWY-TT in TT-unprimed individuals, but suggest that effective TTpriming may be critical to enhance the immune response to subsequent TT conjugate vaccines.

Studies of meningococcal conjugate vaccines specifically evaluating the effects of carrier priming and of prior polysaccharide exposure on subsequent responses to conjugate vaccination are not available. It is not clear whether the results of our study are predictive for individuals living in other countries where TT boosters may be more frequently administered and where fewer subjects may have received prior meningococcal polysaccharide vaccine. It is possible that in these settings higher immune responses to MenACWY-TT would be achieved in older adults. However, this needs to be confirmed in studies conducted outside of the Middle East. Antibody persistence and the response to subsequent MenACWY-TT doses in the elderly warrant further investigation. It would also be informative to study the immunogenicity of TT boosters in older Lebanese adults given the relatively poor response to the TT component of MenACWY-TT observed in this study.

Both of the meningococcal vaccines were associated with very low rates of local and general symptoms. This is consistent with other studies conducted in older individuals where substantially lower reactogenicity has been reported compared to administration of the same vaccines to younger age groups [45].

This study was potentially limited by its open design and because numerous exploratory statistical comparisons were performed without adjustment for multiplicity. Therefore, statistical findings should be interpreted with caution. Inclusion of adults who had previously received meningococcal vaccine was another potential limitation, although we performed sub-analyses to attempt to account for any effects of prior vaccination on the immune response.

\section{Conclusion}

This study provides the first information on MenACWY-TT immunogenicity and safety in the elderly. In adults 56 years of age and older with a varied meningococcal immunization history, MenACWY-TT was immunogenic, with $93 \%$ of subjects achieving rSBA titers well above the seroprotective threshold $(\geq 1: 128)$ against all four serogroups after a single dose. MenACWY-TT was associated with few minor adverse events.

NIMENRIX and MENCEVAX are trademarks of the GlaxoSmithKline group of companies.

Acknowledgements The authors are indebted to the study participants, clinicians, nurses and laboratory technicians who were involved in the study. We acknowledge the contributions of Carelle Tabet, Hiam Naamani and Ahmad Husari, who were involved in this study. The authors also thank Sameh Anis, Nada Riachi, Valerie Sengers for study coordination, Pascal Lestrate for serological analyses, Anne Sumbul for performing the statistical analysis and Leentje Moerman, Archana Jastorff and Ilse Vanhevel for clinical report writing, Dr. Joanne Wolter (on behalf of GlaxoSmithKline Biologicals SA) for preparation of the first draft of the manuscript and Dr. Wouter Houthoofd (Xpe Pharma \& Science) for editorial assistance on behalf of GlaxoSmithKline Biologicals SA.

Sources of support GlaxoSmithKline Biologicals SA was the funding source and was involved in all stages of the study conduct and analysis. GlaxoSmithKline Biologicals SA also funded all costs associated with the development and the publishing of the present manuscript. The corresponding author had full access to the data and was responsible for submission of the publication.

Competing interests GD has received consulting fees and honoraria from GlaxoSmithKline within the last 3 years. NEA, SG and FH have no competing interests. VB, JM and NM are employees of GlaxoSmithKline Vaccines SA. JM and NM report ownership of GlaxoSmithKline stocks and stock options.

Authors contributions Ghassan Dbaibo, Nabil El-Ayoubi, Soha Ghanem and Farah Hajar contributed to the conception and design of the study, the identification of recruitment centers and the acquisition of data. Veronique Bianco was involved in the design and execution of statistical analyses. Jacqueline Miller and Narcisa Mesaros contributed to the conception and coordination of the study, in the analysis and interpretation of results. All authors were involved in the critical review of the manuscript and the approval of its final content.

Open Access This article is distributed under the terms of the Creative Commons Attribution Noncommercial License which permits any noncommercial use, distribution, and reproduction in any medium, provided the original author(s) and the source are credited.

\section{References}

1. Pollard AJ. Global epidemiology of meningococcal disease and vaccine efficacy. Pediatr Infect Dis J. 2004;23(12 Suppl): S274-279.

2. Cohn AC, MacNeil JR, Harrison LH, et al. Changes in Neisseria meningitidis disease epidemiology in the United States, 1998-2007: implications for prevention of meningococcal disease. Clin Infect Dis. 2010;50(2):184-91.

3. Australian Meningococcal Surveillance Programme. Australian Meningococcal Surveillance Programme annual report, 2010. Commun Dis Intell. 2011;35(3):217-28.

4. Gray SJ, Trotter CL, Ramsay ME, et al. Epidemiology of meningococcal disease in England and Wales 1993/94 to 2003/04: contribution and experiences of the Meningococcal Reference Unit. J Med Microbiol. 2006;55(7):887-96. 
5. Harrison LH, Trotter CL, Ramsay ME. Global epidemiology of meningococcal disease. Vaccine. 2009;24(27 Suppl 2):B51-63.

6. Gagneux SP, Hodgson A, Smith TA, et al. Prospective study of a serogroup X Neisseria meningitidis outbreak in northern Ghana. J Infect Dis. 2002;185(5):618-26.

7. Boisier P, Nicolas P, Djibo S, et al. Meningococcal meningitis: unprecedented incidence of serogroup X-related cases in 2006 in Niger. Clin Infect Dis. 2007;44(5):657-63.

8. Decosas J, Koama J-BT. Chronicle of an outbreak foretold: meningococcal meningitis W135 in Burkina Faso. Lancet Infect Dis. 2002;2(12):763-5.

9. Collard JM, Maman Z, Yacouba H, et al. Increase in Neisseria meningitidis serogroup W135, Niger, 2010. Emerg Infect Dis. 2010;16(9):1496-8.

10. Memish ZA, Goubeaud A, Bröker M, et al. Invasive meningococcal disease and travel. J Infect Public Health. 2010;3(4): 143-51.

11. Wilder-Smith A, Goh KT, Barkham T, et al. Hajj-associated outbreak strain of Neisseria meningitidis serogroup W135: estimates of the attack rate in a defined population and the risk of invasive disease developing in carriers. Clin Infect Dis. 2003; 36(6):679-83.

12. Aguilera J-F, Perrocheau A, Meffre C, et al. Outbreak of serogroup W135 meningococcal disease after the Hajj pilgrimage, Europe, 2000. Emerg Infect Dis. 2002;8(8):761-7.

13. Parent du Chatelet I, Barboza P, Taha M. W135 invasive meningococcal infections imported from Sub-Saharan Africa to France, January to April 2012. Euro Surveill. 2012;17(21):1-2.

14. Saudi Ministry of Health Requirements - Hajj. 2012 Jan 9 [online]. Available from URL: http://www.hajinformation.com/ main/p3001.htm.

15. Harrison LH. Prospects for vaccine prevention of meningococcal infection. Clin Microbiol Rev. 2006;19(1):142-64.

16. Poolman J, Borrow R. Hyporesponsiveness and its clinical implications after vaccination with polysaccharide or glycoconjugate vaccines. Expert Rev Vaccines. 2011;10(3):307-22.

17. Granoff DM, Gupta RK, Belshe RB, et al. Induction of immunologic refractoriness in adults by meningococcal $\mathrm{C}$ polysaccharide vaccination. J Infect Dis. 1998;178(3):870-4.

18. Auckland C, Gray S, Borrow R, et al. Clinical and immunologic risk factors for meningococcal $\mathrm{C}$ conjugate vaccine failure in the United Kingdom. J Infect Dis. 2006;194(12):1745-52.

19. Trotter CL, Maiden MCJ. Meningococcal vaccines and herd immunity: lessons learned from serogroup $\mathrm{C}$ conjugate vaccination programs. Expert Rev Vaccines. 2009;8(7):851-61.

20. Stamboulian D, Lopardo G, Lopez P, et al. Safety and immunogenicity of an investigational quadrivalent meningococcal CRM (197) conjugate vaccine, MenACWY-CRM, compared with licensed vaccines in adults in Latin America. Int J Infect Dis. 2010;14(10):e868-75.

21. Dbaibo G, Macalalad N, Reyes MRA-DL, et al. The immunogenicity and safety of an investigational meningococcal serogroups $\mathrm{A}, \mathrm{C}, \mathrm{W}-135, \mathrm{Y}$ tetanus toxoid conjugate vaccine (ACWY-TT) compared with a licensed meningococcal tetravalent polysaccharide vaccine: a randomized, controlled non-inferiority study. Hum Vaccin Immunother 2012;8(7):873-80.

22. Reyes MRA-DL, Dimaano E, Macalalad N, et al. The investigational meningococcal serogroups $\mathrm{A}, \mathrm{C}, \mathrm{W}-135, \mathrm{Y}$ tetanus toxoid conjugate vaccine (ACWY-TT) and the seasonal influenza virus vaccine are immunogenic and well-tolerated when co-administered in adults. Hum Vaccin Immunother. 2012;8 (7):881-7.

23. Nimenrix. Meningococcal group A, C, W 135 and Y conjugate vaccine. European Medicines Agency. 2012. 2012 Jun 22 [online]. Available from: http://www.ema.europa.eu/ema/index. jsp?curl=pages $/$ medicines/human/medicines/002226/human med_001548.jsp\&mid=WC0b01ac058001d124.
24. Ostergaard L, Lebacq E, Poolman J, et al. Immunogenicity, reactogenicity and persistence of meningococcal A, C, W-135 and Y-tetanus toxoid candidate conjugate (MenACWY-TT) vaccine formulations in adolescents aged 15-25 years. Vaccine. 2009;27(1):161-8.

25. Knuf M, Kieninger-Baum D, Habermehl P, et al. A dose-range study assessing immunogenicity and safety of one dose of a new candidate meningococcal serogroups A, C, W-135, Y tetanus toxoid conjugate (MenACWY-TT) vaccine administered in the second year of life and in young children. Vaccine. 2010;28(3): 744-53.

26. Baxter R, Baine Y, Ensor K, et al. Immunogenicity and safety of an investigational quadrivalent meningococcal ACWY tetanus toxoid conjugate vaccine in healthy adolescents and young adults 10 to 25 years of age. Pediatr Infect Dis J. 2011;30(3):e41-8.

27. Vesikari T, Karvonen A, Bianco V, et al. Tetravalent meningococcal serogroups A, C, W-135 and Y conjugate vaccine is well tolerated and immunogenic when co-administered with measlesmumps-rubella-varicella vaccine during the second year of life: an open, randomized controlled trial. Vaccine. 2011;29(25): 4274-84.

28. Memish ZA, Dbaibo G, Montellano M, et al. Immunogenicity of a single dose of tetravalent meningococcal serogroups A, C, $\mathrm{W}-135$, and $\mathrm{Y}$ conjugate vaccine administered to 2- to 10-yearolds is noninferior to a licensed-ACWY polysaccharide vaccine with an acceptable safety profile. Pediatr Infect Dis J. 2011;30(4): e56-62.

29. Knuf M, Pantazi-Chatzikonstantinou A, Pfletschinger U, et al. An investigational tetravalent meningococcal serogroups $\mathrm{A}, \mathrm{C}$, $\mathrm{W}-135$ and Y-tetanus toxoid conjugate vaccine co-administered with InfanrixTM hexa is immunogenic, with an acceptable safety profile in 12-23-month-old children. Vaccine. 2011;29(25): 4264-73.

30. Bermal N, Huang L-M, Dubey A, et al. Safety and immunogenicity of a tetravalent meningococcal serogroups A, C, W-135 and $\mathrm{Y}$ conjugate vaccine in adolescents and adults. Hum Vaccine. 2011;7(2):239-47.

31. Maslanka SE, Gheesling LL, Libutti DE, et al. Standardization and a multilaboratory comparison of Neisseria meningitidis serogroup A and C serum bactericidal assays. The Multilaboratory Study Group. Clin Diagn Lab Immunol. 1997;4(2):156-67.

32. Borrow R, Balmer P, Miller E. Meningococcal surrogates of protection-serum bactericidal antibody activity. Vaccine. 2005; 23(17-18):2222-7.

33. Andrews N, Borrow R, Miller E. Validation of serological correlate of protection for meningococcal $\mathrm{C}$ conjugate vaccine by using efficacy estimates from postlicensure surveillance in England. Clin Diagn Lab Immunol. 2003;10(5):780-6.

34. Melville-Smith ME, Seagroatt VA, Watkins JT. A comparison of enzyme-linked immunosorbent assay (ELISA) with the toxin neutralization test in mice as a method for the estimation of tetanus antitoxin in human sera. J Biol Stand. 1983;11(2): 137-44.

35. Jokhdar H, Borrow R, Sultan A, et al. Immunologic hyporesponsiveness to serogroup $\mathrm{C}$ but not serogroup A following repeated meningococcal A/C polysaccharide vaccination in Saudi Arabia. Clin Diagn Lab Immunol. 2004;11(1):83-8.

36. Keyserling H, Papa T, Koranyi K, et al. Safety, immunogenicity, and immune memory of a novel meningococcal (groups A, C, Y, and $\mathrm{W}-135)$ polysaccharide diphtheria toxoid conjugate vaccine (MCV-4) in healthy adolescents. Arch Pediatr Adolesc Med. 2005;159(10):907-13.

37. Al-Mazrou Y, Khalil M, Findlow H, et al. Immunogenicity and safety of a meningococcal quadrivalent conjugate vaccine in Saudi Arabian adolescents previously vaccinated with one dose of bivalent and quadrivalent meningococcal polysaccharide 
vaccine: phase III, controlled, randomized, modified blindobserver study. Clin Vaccine Immunol. 2012;19(7):999-1004.

38. Dbaibo G, Van der Wielen M, Reda M, et al. The tetravalent meningococcal serogroups $\mathrm{A}, \mathrm{C}, \mathrm{W}-135$, and $\mathrm{Y}$ tetanus toxoid conjugate vaccine is immunogenic with a clinically acceptable safety profile in subjects previously vaccinated with a tetravalent polysaccharide vaccine. Int J Infect Dis. 2012;16(8):e608-6.

39. United Nations Population Division. World population prospects: the 2010 revisions population database. 2011 [online]. Available from: http://esa.un.org/unpd/wpp/unpp/panel_indicators.htm.

40. Dagan R, Poolman J, Siegrist C-A. Glycoconjugate vaccines and immune interference: a review. Vaccine. 2010;28(34):5513-23.

41. Karabay O, Ozkardes F, Tamer A, Karaarslan K. Tetanus immunity in nursing home residents of Bolu, Turkey. BMC Public Health. 2005;12(5):5.
42. Redwan E-RM, Al-Awady MK. Prevalence of tetanus immunity in the Egyptian population. Hum Antibodies. 2002;11(1-2):55-9.

43. Cilla G, Sáenz-Domínguez JR, Montes M, Part C, Pérez-Trallero E. Immunity against tetanus in adults over the age of 49 years. Med Clin. 1994;103(15):571-3.

44. Theeten H, Rümke H, Hoppener FJP, et al. Primary vaccination of adults with reduced antigen-content diphtheria-tetanus-acellular pertussis or dTpa-inactivated poliovirus vaccines compared to diphtheria-tetanus-toxoid vaccines. Curr Med Res Opin. 2007; 23(11):2729-39.

45. Van Damme P, McIntyre P, Grimprel E, et al. Immunogenicity of the reduced-antigen-content dTpa vaccine (Boostrix $\left({ }^{\circledR}\right)$ ) in adults 55 years of age and over: a sub-analysis of four trials. Vaccine. 2011;29(35):5932-9. 